\title{
La evaluación de competencias profesionales adquiridas a través de la experiencia y vías no formales de formación
}

\section{Assessment of professional skills acquired through experience and non-formal training routes}

\author{
Manuel Madrid García ${ }^{1}$ \\ manuel.madrid@um.es \\ Juan Carlos Solano Lucas \\ jcsolano@um.es \\ Pedro Baños Páez \\ pbanos@um.es \\ Universidad de Murcia, España
}

\section{Resumen:}

En este artículo hacemos una aproximación a los resultados de una investigación realizada en la Universidad de Murcia sobre los procesos de asesoramiento, evaluación y resultados obtenidos en el Procedimiento de Reconocimiento, Evaluación, Acreditación y Registro de las competencias profesionales (PREAR) para la primera convocatoria de cualificaciones profesionales en la Región de Murcia (Educación Infantil -E.I.-, Atención sociosanitaria a personas en el domicilio -ASPD- y Atención sociosanitaria a personas dependientes en instituciones sociales-ASPDI-).

La hipótesis de partida, que sirvió de marco de referencia para este estudio, se centra en la idea de que para los candidatos de este proceso es más determinante, en lo que se

\begin{abstract}
:
This article discusses the results of a study conducted at the University of Murcia on the processes of counseling, evaluation and results of the procedure Recognition, Evaluation, Accreditation and Registration of Professional Skills for the first work selection process aimed at obtaining qualified professional status in the Region of Murcia (infant education, geriatric care to people at home, and geriatric care for dependents in social institutions).

The hypothesis which gave rise to this study focuses on the idea that for the candidates going through this process, when it comes to accrediting work competences, work experience is more important than non-formal training which the candidate might have.
\end{abstract}

1 Dirección para correspondencia (correspondence address):

Manuel Madrid García. Dpto. de Sociología y Trabajo Social. Facultad de Economía y Empresa. Universidad de Murcia. Campus Universitario de Espinardo. 30100 Murcia (España). 
La evaluación de competencias profesionales adquiridas a través de la experiencia y vías no formales de formación

Manuel Madrid García, Juan Carlos Solano lucas y Pedro Baños Páez

refiere a conseguir la acreditación de competencias, la propia experiencia laboral, que la formación no formal alcanzada.

Esta investigación se realizó sobre el registro de los 450 candidatos/as que participaron en el PREAR (150 en cada una de las tres cualificaciones convocadas) y a un número representativo del personal habilitado como asesor y/o evaluador que también intervino en este procedimiento. Dicho trabajo hizo uso de las fuentes primarias de datos del Instituto de las Cualificaciones de la Región de Murcia (ICUAM) sobre candidatos y asesores/ evaluadores que habían participado en el procedimiento. También se realizaron distintas entrevistas a informantes clave y se organizaron varios grupos de discusión en torno a participantes en los procesos para dichas cualificaciones profesionales.

\section{Palabras clave:}

Sociología de las Cualificaciones Profesionales, Competencia Profesional, Reconocimiento, Evaluación, Acreditación, Experiencia Profesional, Formación No Formal.
The research was conducted with 450 candidates who participated in the selection process (150 in each of the three specialities) and a representative number of counsellor/evaluator-qualified staff who also participated in this procedure.

This work used the primary data sources from the Institute of Qualifications of the Region of Murcia (ICUAM) about candidates and assessors/evaluators who had participated in the procedure. Several interviews with key informants were also conducted and various discussion groups with participant in the selection process were organised.

\section{Key words:}

Sociology of Professional Qualifications; Professional Competence; Recognition; Evaluation; Accreditation; Professional Experience; Non-Formal Education.

\section{Résumé:}

Dans cet article nous faisons d'une approximation des résultats des recherches menées dans l'Université de Murcie sur les processus de consultation, d'évaluation et les résultats de la procédure de reconnaissance, l'évaluation, I'accréditation et l'enregistrement des compétences professionnelles (PREAR) pour le premier appel de qualification professionnelle dans la Région de Murcie (Education Infantile -El-, les soins gériatriques de personnes au domicile -ASPD- et les soins gériatriques aux personnes dépendantes dans les institutions sociales -ASPDI).

L'hypothèse qui a été le cadre de la présente étude est fondée sur l'idée que pour les candidats de ce processus est plus décisif, en ce qui concerne la réalisation réussie de l'accréditation des compétences, la formation informelle qu'ils ont réalisée.

Cette recherche a été menée sur le dossier des 450 candidats / ayant participé à la PREAR (150 dans chacune des trois qualifications appelés) et un nombre représentatif d'un personnel qualifié en tant que conseiller et / ou évaluateur qui a également participé à cette procédure. Ce travail a fait usage des sources primaires de données des qualifications de la Région de Murcie (ICUAM) sur les candidats et les évaluateurs qui ont participé à la procédure. On a également mené plusieurs entretiens avec des informateurs clés et les divers groupes de discussion pour les qualifications professionnelles.

\section{Mots clés:}

Sociologie des qualifications professionnelles; la compétence professionnelle; la reconnaissance; I'évaluation et l'accréditation; I'expérience professionnelle; l'éducation non formelle.

Fecha de recepción: 8-10-2013

Fecha de aceptación: 21-11-2015 


\section{Introducción}

Como respuesta a las nuevas exigencias del mercado laboral europeo, en la Cumbre de la Unión Europea celebrada el año 2000 en Lisboa se plantearon los desafíos de la nueva economía basada en el conocimiento.

En la Resolución del Consejo Europeo sobre educación permanente ${ }^{2}$ se señalaba la prioridad de reconocer y validar entre países y sectores, las cualificaciones resultantes de los aprendizajes formal, no formal e informal, instando a la eliminación de barreras entre estas formas de aprendizaje.

En la Declaración de Copenhague ${ }^{3}$ se busca dar prioridad a los procesos de reconocimiento de las competencias y de las cualificaciones mediante una cooperación reforzada en el área de la Formación Profesional.

En este contexto se plantean "nuevos conceptos de producción que Ilevan asociada una demanda de nuevos requerimientos de cualificación en los puestos de trabajo, siendo las principales características de las cualificaciones demandadas: capacidad de abstracción, autonomía de los puestos de trabajo, inteligencia emocional, polivalencia y capacidad de aprendizaje continuo". (Köhlerg y Martín, 2007: 365).

Entre los hitos fundamentales que han marcado este proceso en España destaca la Ley Orgánica 5/2002 de las Cualificaciones y de la Formación Profesional ${ }^{4}$, pues establece la creación del Sistema Nacional de Cualificaciones; se configura así un nuevo Sistema de Formación Profesional en el que las cualificaciones profesionales son acreditables por cuatro vías:

- Formación Profesional Inicial, impartida en el sistema educativo.

- Formación Profesional para el Empleo (subsistema que integra la Formación Ocupacional y la Formación Continua)

- Vías no formales de formación.

- Experiencia laboral.

Estas dos últimas vías son las que determinan el ámbito de estudio de la presente investigación, ya que son el marco de referencia del PREAR 5 .

2 Resolución 2002/C 163/01. Diario Oficial de las Comunidades Europeas de 9/07/2002.

3 Declaración de Copenhague, Noviembre 2002.

4 Publicada en BOE núm. 147 de 20 de Junio de 2002.

5 Este procedimiento queda regulado en el Real Decreto 1224/2009, de 17 de julio, de reconocimiento de las competencias profesionales adquiridas por experiencia laboral. 
La evaluación de competencias profesionales adquiridas a través de la experiencia y vías no formales de formación

Manuel Madrid García, Juan Carlos Solano lucas y Pedro Baños Páez

En la puesta en marcha en la Región de Murcia del PREAR con las cualificaciones $^{6}$ de E.I., ASPD y ASPDI, se vio conveniente realizar un estudio que permitiera analizar todo el proceso, desde su inicio hasta la obtención de las primeras acreditaciones.

En este artículo se presentan algunas aportaciones a esta temática, en concreto a la primera convocatoria del PREAR en la Región de Murcia para esas tres cualificaciones profesionales, abarcando desde una aproximación a los solicitantes potenciales del PREAR, hasta el diseño y puesta en marcha de un proyecto de investigación para el seguimiento de este proceso de acreditación?

Uno de los objetivos de este proyecto es servir de base a futuros estudios comparativos de las vías de adquisición y reconocimiento de estas cualificaciones profesionales en distintas Comunidades Autónomas, incluyendo la perspectiva cualitativa (valoraciones de los agentes implicados en este procedimiento) con el fin de ajustar el proceso a las necesidades de los perfiles profesionales actuales, cuyas competencias adquiridas por la experiencia y vías no formales de formación son evaluadas.

Otra finalidad de este estudio es establecer una referencia a la hora de realizar nuevas convocatorias de acreditación oficial de cualificaciones profesionales ya que sus conclusiones pueden ser tenidas en cuenta por los organismos públicos competentes con la finalidad de mejorar la eficiencia de dichas convocatorias.

Respecto a la realización de estudios previos sobre la valoración del PREAR, considerando también aportaciones cualitativas de los agentes implicados, hay que señalar que no existen investigaciones de este tipo a nivel nacional. Lo que se ha realizado más frecuentemente en los últimos años son estudios cuantitativos sobre resultados obtenidos tras este procedimiento por cualificación profesional a nivel de cada Comunidad Autónoma, como es en el caso de la Región de

6 Orden 1/07/2010 de Convocatoria en la Región de Murcia del PREAR para las cualificaciones de: Atención sociosanitaria a personas en instituciones sociales, Atención sociosanitaria a personas en el domicilio y Educación Infantil.

7 Proyecto: Investigación sobre los procesos de Asesoramiento, Evaluación y Resultados obtenidos en el PREAR para las cualificaciones convocadas en la CARM, aprobado en Mayo de 2011 por la Comisión de Seguimiento del Convenio de cooperación en materia de formación inicial y permanente del profesorado que ejerce en los niveles anteriores a la Universidad entre la Consejería de Educación, Formación y Empleo de la CARM y la Universidad de Murcia, en el marco del Programa III del Convenio. 
Murcia $^{8}$. También cabe destacar los primeros estudios de resultados publicados por el Instituto Nacional de las Cualificaciones ${ }^{9}$.

La base del proceso de certificación establecido en relación a los conocimientos adquiridos por la experiencia y por vías no formales de formación, tiene como referente los conceptos de cualificación y de competencia. Por la relevancia que poseen estos conceptos, a continuación se expone una síntesis de su marco teórico y conceptual en el sistema de la Formación Profesional actual.

\section{Cualificaciones y competencias profesionales: enfoques teóricos y marco conceptual}

\section{1-La cualificación profesional: una aproximación a su marco conceptual}

En el actual sistema de la Formación Profesional las competencias profesionales se han organizado por cualificaciones ${ }^{10}$.

En España las cualificaciones profesionales se organizan en el Sistema Nacional de Cualificaciones y Formación Profesional ${ }^{11}$, y en el Catálogo Nacional de Cualificaciones Profesionales ${ }^{12}$.

8 Instituto de las Cualificaciones de la Región de Murcia. Resultados y estadísticas de las convocatorias PREAR 2010, 2012, 2013. Publicado en: http://www.icuam.es/web/ guest/91

9 Instituto Nacional de las Cualificaciones. Datos recogidos sobre el Procedimiento de Reconocimiento de Competencias adquiridas por experiencia laboral. Agosto 2013. Publicado en: http://www.educacion.gob.es/educa/incual/pdf/Acreditacion/Inf_Datos_Proc_Acred_2013_agosto.pdf

10 La Ley Orgánica 5/2002, de 19 de junio, de las cualificaciones y la formación profesional define la cualificación profesional como el conjunto de competencias profesionales con significación para el empleo que pueden ser adquiridas mediante formación modular u otros tipos de formación y a través de la experiencia laboral.

11 Conjunto de instrumentos y acciones necesarios para promover y desarrollar la integración de las ofertas de la formación profesional. Su objetivo es promover y desarroIlar la evaluación y acreditación de las correspondientes competencias profesionales, de forma que se favorezca el desarrollo profesional y social de las personas y se cubran las necesidades del sistema productivo.

12 Instrumento del Sistema Nacional de las Cualificaciones y Formación Profesional (SNCFP) que ordena las cualificaciones profesionales susceptibles de reconocimiento y acreditación, identificadas en el sistema productivo en función de las competencias apropiadas para el ejercicio profesional. Comprende las cualificaciones profesionales más significativas del sistema productivo español, organizadas en familias profesio- 
En este Catálogo se especifican las cualificaciones profesionales desde el nivel 1 al 5, que tienen su correspondencia con los niveles del 1 al 8 del Marco Europeo de Cualificaciones (EQF) (Ver tabla 1).

La investigación que nos ocupa se ha centrado en dos cualificaciones profesionales de nivel 2 (ASPD y ASPDI) y en una de nivel 3 (EI) para el Catálogo Nacional de Cualificaciones Profesionales CNCP.

Tabla 1. Cualificaciones Profesionales en el EQF y en el CNCP.

\begin{tabular}{|c|c|c|}
\hline EQF & CNCP & Acreditación \\
\hline Nivel 1 & \multirow[t]{2}{*}{ Nivel 1} & \multirow[t]{2}{*}{ Operario } \\
\hline Nivel 2 & & \\
\hline Nivel 3 & \multirow[t]{2}{*}{ Nivel 2} & \multirow[t]{2}{*}{ Técnico Medio } \\
\hline Nivel 4 & & \\
\hline Nivel 5 & Nivel 3 & Técnico Superior \\
\hline Nivel 6 & Nivel 4 & Grado \\
\hline Nivel 7 & Nivel 5 & Máster \\
\hline Nivel 8 & Sin definir & Doctor \\
\hline
\end{tabular}

Fuente: INCUAL: https://www.educacion.gob.es/iceextranet/. Consulta: 10/06/2013

Las cualificaciones se ordenan y se agrupan en diferentes familias profesionales y en los cinco niveles de Cualificación Profesional en el Sistema Nacional de Cualificaciones. Estos niveles se determinan según el grado de complejidad, autonomía y responsabilidad necesarias para realizar una actividad laboral. A mayor nivel de cualificación se asocia una mayor categoría profesional, capacidad de trabajo y especialización del individuo en el puesto de trabajo ${ }^{13}$.

A cada cualificación se le asigna una serie de elementos: una competencia general, entorno profesional, sectores productivos y ocupaciones y puestos de trabajo a los que permite acceder.

Cada cualificación engloba diferentes unidades de competencia, a cada una de las cuales se asocia la formación necesaria a adquirir mediante diferentes módulos formativos.

El concepto de cualificación se operativiza en el concepto de compe-

nales y niveles. Constituye la base para elaborar la oferta formativa de los títulos y los certificados de profesionalidad.

13 Las cualificaciones convocadas en el PREAR en la Región de Murcia son de los niveles II y III.

NIVEL II: Atención sociosanitaria a personas en instituciones sociales y Atención sociosanitaria a personas en el domicilio. NIVEL III: Educación Infantil. 
tencia profesional ${ }^{14}$ y de esta forma queda estructurado en el propio sistema de la Formación Profesional. A continuación exponemos una breve aproximación a los diferentes enfoques teóricos sobre este término clave.

\section{2- La competencia profesional: principales enfoques teóricos}

El concepto de competencia profesional tiene su origen en trabajos de la psicología industrial y organizacional norteamericana de finales de la década de 1960 y principios de 1970 (Spencer el al., 1993).

Desde esta época, el interés en las organizaciones se ha ido trasladando desde los puestos de trabajo, como elemento fundamental, a las personas y, en particular, a las competencias que éstas aportan y su relación con las que demanda cada puesto de trabajo.

Un aspecto que debe introducirse en el estudio de las competencias es lo que se ha denominado como conocimiento tácito o cualificaciones tácitas (Wood, 1987) que se refiere a aquellos conocimientos, actitudes o competencias que se adquieren mediante el proceso general de socialización, la influencia del entorno social o las socializaciones diferenciadas por razón de género, clase social, edad u origen étnico. Este tipo de competencias no suelen ser detectadas ni analizadas en los procesos habituales de evaluación. Wood también señala la importancia de la construcción social de las cualificaciones que no proceden, por tanto, de tecnologías ajenas a la acción de los agentes sociales (Wood, 1981).

También conviene atender a la dimensión de género en este marco ya que se considera que el trabajo «refleja los supuestos talentos de las mujeres, antes que una cualificación adquirida para la cual sería apropiado un reconocimiento salarial o social», tal como señala Jenson (1989: 151).

En la mayoría de aportaciones, a partir de la década de 1990, se considera que los términos de Cualificación y Competencia están relacionados. Alex (1991) afirma que si la cualificación responde a una dimensión personal, la competencia profesional forma parte de ella y responde a una dimensión social. Alaluf y Stroobants (1994) también señalan cómo

14 La Ley Orgánica 5/2002, de 19 de junio, de las cualificaciones y la formación profesional define la competencia profesional como el conjunto de conocimientos y capacidades que permitan el ejercicio de la actividad profesional conforme a las exigencias de la producción y el empleo. Cabe mencionar que autores como Olaz (2011) añade una tercera dimensión (las habilidades) a este concepto, si bien señala que algunas habilidades pueden verse a menudo solapadas con las capacidades. 
la competencia profesional forma parte de la cualificación y cómo sirve para demostrarla o ponerla a prueba.

Atendiendo a su valor de cara al desempeño laboral, la competencia se ha definido como una característica subyacente en una persona, que está causalmente relacionada con una actuación exitosa en el desempeño en un puesto de trabajo (Boyatzis, 1982).

Tomando en consideración los componentes de la competencia, Le Boterf (2001) la define como una construcción a partir de una combinación de recursos individuales (conocimiento, saber hacer, cualidades o aptitudes), y recursos del ambiente (relaciones, documentos, informaciones y otros) que son movilizados para lograr un desempeño.

Cabe precisar que existe una corriente crítica respecto a la utilización de este concepto ya que "distorsiona tanto la información sobre las competencias de las personas como la del funcionamiento del mercado de trabajo y los mecanismos que lo regulan" (Planas et al., 2000).

Por ello conviene destacar que se ha terminado implantando una noción adecuacionista de las competencias, frente a la noción genuina de las mismas y por ello "las competencias no son relativas a los individuos y a sus itinerarios particulares de educación formal, no-formal e informal, considerados globalmente, sino que están relacionadas con ciclos particulares de educación formal cuyas competencias asociadas están definidas previa y meticulosamente" (Planas, 2013: 89).

En el ámbito de la Formación Profesional que es en el que se centra el presente estudio, tiene especial relevancia la aportación de Oriol Homs que señala tres posibles interpretaciones de la competencia. La primera la concibe "como la iniciativa y la responsabilidad que el individuo asume ante situaciones profesionales a las que se enfrenta" (Homs, 2008: 140). La segunda se centra en "la inteligencia práctica de las situaciones, la cual se basa en los conocimientos adquiridos y los transforma a medida que se enfrenta ante diversidad de situaciones" (Homs, 2008: 141). Finalmente, la tercera se refiere al trabajo colectivo "en torno de una misma situación, de compartir los retos y de asumir las áreas de corresponsabilidad". Este tercer enfoque aporta la dimensión colectiva inherente a todo proceso de trabajo. Son los individuos quienes movilizan las competencias, pero "se requiere la competencia colectiva de una red de actores para conseguir un resultado satisfactorio" (Homs, 2008: 141).

También se ha analizado la importancia de las cualificaciones y el 
papel que éstas juegan en la realidad social, no sólo la faceta educativa, sino también la económica y la laboral, en las distintas sociedades.

Desde la perspectiva Funcionalista, especialmente a través de la Teoría del Capital Humano (Schultz 1961, Becker 1964), se entiende que la competencia profesional es un atributo susceptible de medición cuantitativa y que tiene un carácter objetivo, independiente del observador. Así, buscan establecer medidas cuantitativas de las competencias profesionales que se ajusten a sus planteamientos metodológicos. Estas medidas deben representar de forma significativa la variedad de las diversas competencias en el marco laboral. Para hacer esto, la medida llega a hacerse frecuentemente muy abstracta, aunque su gran nivel de abstracción no garantiza necesariamente mediciones rigurosas, pudiendo afectar con ello su aceptación.

Los sociólogos que siguen este planteamiento, tienden a abordar la competencia profesional como un atributo de los trabajos, antes que de las personas. Muchos se han centrado en tres medidas principales del DOT ${ }^{15}$ : sobre la complejidad de los datos, las personas y del equipamiento; o combinación de estas tres en una única medida Ilamada "complejidad global del trabajo" (Kohn y Schooler, 1983).

Desde un enfoque neoweberiano se intentan comprender, por un lado, las condiciones por las que las ocupaciones son socialmente delimitadas, construidas, y por otro, los procesos mediante los cuales algunos trabajos Ilegan a merecer un estatus más alto que otros. El método más directo de realzar el poder de una ocupación suele ser el alejarlo de la competición del mercado, lo que Weber y otros Ilaman "cierre social" (Parkin, 1979) restringiendo la entrada a esa ocupación, demandando largos períodos previos de aprendizaje, etc.; de esta forma, no sólo se consiguen recompensas económicas para sus integrantes, sino que también dicha ocupación eleva su competencia y estatus, alentando una percepción social de que precisa conocimiento y preparación excepcionales, sin que los títulos o certificados vengan a garantizar realmente las competencias del poseedor (Collins, 1976). Se entiende, por tanto, que las ocupaciones con ingresos y prestigio social más altos lleven a cabo una labor más cualificada. Los elementos ideológicos que reclaman la importancia social y la destreza observada de la ocupación, son normalmente entendidos como dependientes de la capacidad previa de la ocupación para protegerse a sí misma de la competición del mercado y controlar la formación y la socialización en la ocupación.

15 Diccionario de títulos profesionales del Ministerio de Trabajo norteamericano, 1965. 
Otra aportación próxima a los planteamientos neoweberianos es la relativa a la Etnometodología, que se plantea una perspectiva de la actividad humana -y de la competencia profesional- que trata de evidenciar los problemas epistemológicos que hacen difícil cuantificar la competencia desde un punto de vista positivista.

Desde la perspectiva marxiana se consideró el trabajo cualificado frente al no cualificado, argumentando que el valor del poder del trabajo (un salario) es básicamente el coste de reproducir y mantener a los trabajadores. El salario superior del trabajo especializado reflejaría el pago por los costes de la puesta en valor de tales competencias. En este marco teórico se emplea una noción clara de competencia como conjunto de habilidades superiores desarrolladas con un proceso formativo especial.

El aspecto construccionista social de la competencia profesional dentro del marxismo se confirma con la descripción de una realidad; la de que la posición social y la competencia percibida de una ocupación proceden, en gran medida, del poder de los trabajadores en el contexto social correspondiente, antes que de la complejidad intrínseca de la tarea en sí misma.

En el marco de la perspectiva de la alienación de Marx, es un rasgo central a estudio la transición histórica desde el trabajo que está bajo el propio control del trabajador, al trabajo que es dirigido por una administración. De esta forma se añade un elemento distintivo al concepto marxista de competencia, el control. Por ello, el lenguaje de la alienación (pérdida de control) se entrelaza con el lenguaje de la descualificación. Así, un trabajador especializado o alguien que lleva a cabo una rutina establecida por otros es un "no cualificado" (Braverman, 1974).

Otra aportación interesante en este mismo marco teórico es la formulada por Planas que afirma que "Las competencias adquiridas informalmente solían ser reconocidas tácitamente por las empresas (en el proceso de contratación y promoción). Ahora pasan a ser certificadas por instancias educativas y gubernamentales, lo cual no se corresponde con los usos y costumbres del mercado de trabajo" (Planas, 2013: 85). De este modo se consolidaría un reconocimiento formal (ejercido por el sistema educativo) que complementa al reconocimiento tácito en el ámbito laboral.

En la perspectiva neomarxiana se parte de la concepción de que competencia profesional y autonomía en el desempeño de tareas laborales, a menudo, van empíricamente unidas; sin embargo, hacer del control, la 
autodirección y la competencia profesional elementos equivalentes, puede llevar a confusiones. De esta forma, autores como Crompton y Jones (1984) señalan que si el trabajador no decide qué herramientas o métodos emplear para efectuar una tarea y si no puede programar qué hacer y cuándo, carece no solo de control sino también de competencia.

Esta vinculación entre control y competencia profesional tiene especial importancia para el estudio de un proceso como el PREAR, ya que el nivel competencial que se verifique y acredite a los trabajadores tendrá que basarse en la existencia de un control y autodirección ${ }^{16}$ que han debido tener en sus puestos de trabajo. Desde este planteamiento, parece evidente que acreditar un tiempo de experiencia profesional a través de contratos de trabajo y/o certificaciones de cotización laboral y/o una asistencia a actividades formativas no formales no deben ser considerados garantía de poseer una competencia profesional; aunque sirvan para establecer finalmente quiénes pueden participar en un proceso de acreditación y reconocimiento de competencias profesionales.

\section{Evaluación del proceso y resultados del PREAR en Murcia}

En el marco del Proyecto: Investigación sobre los procesos de Asesoramiento, Evaluación y Resultados obtenidos en el PREAR para las cualificaciones convocadas en la CARM, se ha realizado el seguimiento del proceso y resultados del PREAR en la Región de Murcia para las tres cualificaciones convocadas en el año 2010 y concluidas en mayo de 2012.

Se debe tener en cuenta que las dos primeras cualificaciones convocadas (Atención sociosanitaria a personas dependientes en instituciones sociales y Atención sociosanitaria a personas en el domicilio) son de nivel 2, tomando como referencia el Marco Europeo de Cualificaciones, mientras que la de Educación Infantil es de nivel 3; con lo que el proceso de seguimiento de esta última ha sido más complejo al poseer mayor número de unidades de competencia a reconocer en este PREAR.

Este Procedimiento buscaba el reconocimiento de la experiencia y de la formación no formal a efectos de la acreditación de las diferentes Unidades de Competencia (UUCC) de cada una de las cualificaciones convocadas.

16 Existen diferentes niveles de autodirección dependiendo de los niveles de cualificación profesional, siendo ésta más alta cuanto más elevados son dichos niveles. 
Uno de los motivos de convocar las dos cualificaciones profesionales de nivel 2 fue lo establecido en la Resolución de 2 de diciembre de 2008, de la Secretaría de Estado de Política Social, Familias y Atención a la Dependencia y a la Discapacidad, que establece la obligatoriedad de disponer del certificado de profesionalidad para los trabajadores de este sector para el año 2015; dicho certificado se puede obtener acreditando previamente todas las Unidades de Competencia de la cualificación profesional a través del propio PREAR.

Los objetivos específicos e hipótesis ${ }^{17}$ que han guiado el desarrollo del proyecto de investigación ${ }^{18}$ han servido para orientar el seguimiento del PREAR y la evaluación de sus resultados.

Se ha diseñado un modelo de evaluación y análisis a través de una triangulación metodológica.

Se han determinado los actores clave para ser entrevistados o para participar en grupos de discusión. Se ha mantenido una coordinación contínua con el ICUAM (Instituto de las Cualificaciones de la Región de Murcia) para conseguir el acceso a las bases de datos de los participantes en el PREAR (asesores, evaluadores y candidatos).

Se ha realizado una explotación estadística de los datos procedentes del ICUAM sobre candidatos y asesores/evaluadores que han participado en el procedimiento.

Se han realizado cuatro entrevistas en profundidad: una a la coordinadora del SEF (Servicio de Empleo y Formación) para conocer el proceso

17 OBJETIVOS ESPECÍFICOS:

- Valorar el proceso de asesoramiento seguido con cada una de las tres cualificaciones convocadas.

- Valorar el proceso de evaluación seguido con cada una de las tres cualificaciones convocadas.

-Realizar una primera valoración general de resultados del PREAR en cada una de las tres cualificaciones.

-Realizar una primera comparativa sobre la acreditación de competencias realizada por vías no formales de formación y por Experiencia laboral.

HIPÓTESIS:

-El proceso de asesoramiento seguido para las tres cualificaciones se valora como ajustado a unos stándares de eficiencia/eficacia predefinidos.

-El proceso de evaluación seguido para las tres cualificaciones se valora como ajustado a unos stándares de eficiencia/eficacia predefinidos.

-Los resultados en términos de competencias acreditadas se valoran como conseguidos a un mayor nivel por los solicitantes con mayor puntuación en experiencia laboral que en vías no formales de formación.

18 Ver nota al pié $n^{\circ} 6$. 
previo de información y asesoramiento sobre el PREAR realizado desde ese Servicio; otra a un evaluador de la cualificación de Atención sociosanitaria a personas dependientes en instituciones sociales para completar la información obtenida con el grupo de discusión de esta cualificación; otras dos entrevistas realizadas a una asesora y a una evaluadora de la cualificación de Educación infantil; ya que para esta última cualificación, no ha sido posible desarrollar los Grupos de Discusión (GGDD), ni para asesores/evaluadores ni para candidatos.

Se han realizado cuatro grupos de discusión para las dos cualificaciones de nivel 2, uno para candidatos y otro para asesores/evaluadores por cada una de éstas.

Para el diseño y desarrollo de los GGDD se ha seguido el siguiente proceso:

$1^{\circ}$.- Diseño de contenidos de los GGDD: Universidad de Murcia (Departamento de Sociología y Política Social y Departamento de Teoría e Historia de la Educación, vinculados al proyecto de investigación, en coordinación con ICUAM para determinar dichos contenidos partiendo de la información que se ha facilitado desde este Instituto, incluyendo algunas variables que les interesa conocer.

$2^{\circ}$.- Preselección de participantes: Universidad de Murcia, partiendo de las bases de datos que ha facilitado el ICUAM sobre los evaluadores, asesores y candidatos que han participado en cada una de las tres cualificaciones.

Los criterios utilizados en la selección de los participantes en GGDD se han basado en la representatividad estructural de los perfiles intragrupales de los candidatos evaluados, así como de los asesores y evaluadores del PREAR. Para ello se han explotado las distintas bases de datos suministrados por el ICUAM.

$3^{\circ}$.- Selección de candidatos, por parte del ICUAM para confirmar su conformidad para cesión de datos personales a la Universidad de Murcia.

$4^{\circ}$.- Acceso a datos personales y citación: Universidad de Murcia.

$5^{\circ}$.- Desarrollo de los GGDD.

$6^{\circ}$.- Análisis de los contenidos.

El proceso comenzó con la entrevista a la coordinadora del SEF (Servicio de Empleo y Formación de la Región de Murcia) ya que fue este Servicio 
el que llevó a cabo el proceso de información y asesoramiento a los potenciales solicitantes, con anterioridad al inicio del PREAR.

En el análisis de los datos obtenidos sobre este procedimiento hay que señalar que en esta primera convocatoria de 2010 el número límite de candidatos aceptados fue de 150 para cada una de las tres cualificaciones.

Respecto a la primera cualificación: Atención sociosanitaria a personas dependientes en instituciones sociales (ASPDI), en la figura 1 se puede constatar el alto número de solicitantes y que un alto porcentaje de los que pasan a la fase de Evaluación Ilegan a acreditar todas las Unidades de Competencia. También es destacable que la gran mayoría de candidatos fueron mujeres.

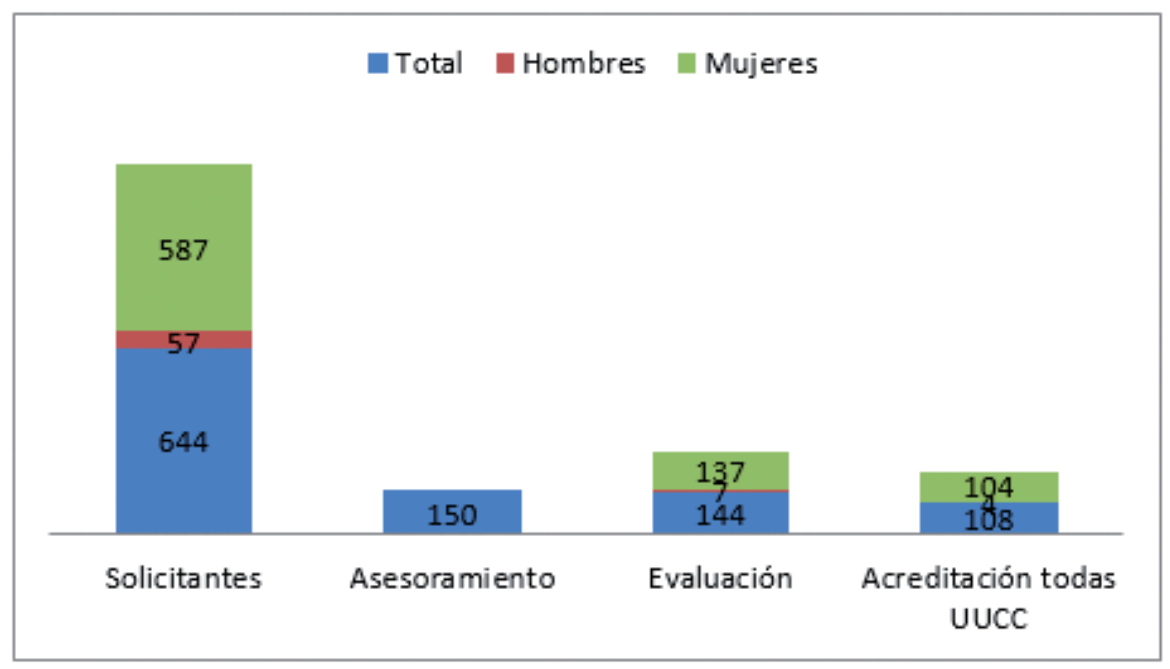

Figura 1. Participación en el PREAR para la cualificación de ASPDI en la Región de Murcia. Convocatoria 2010.

Fuente: Elaboración propia a partir de la explotación de datos facilitada por el ICUAM.

\section{Resultados:}

Opinión de asesores/evaluadores:

En relación a:

Fase de habilitación como asesor/evaluador: Esta fase fue valorada en general como positiva, aunque se debería haber planteado desde una metodología más práctica, incluyendo una formación procedimental en el manejo de instrumentos específicos de asesoramiento y de evaluación. 
Desarrollo de los procesos de asesoramiento y de evaluación: En general se valoró que el calendario se alargó por la dificultad de compaginar los turnos de trabajo de los habilitados (asesores / evaluadores) con los de los candidatos.

Se planteó la necesidad de contar con la figura de un coordinador entre asesores y evaluadores para facilitar un trabajo conjunto.

Perfil de los solicitantes atendidos: En su mayoría se trataba de trabajadoras procedentes de residencias o de centros ocupacionales con amplia experiencia y también formación no formal referida a su perfil profesional (cursos, seminarios, etc.).

Evaluación de los itinerarios, formativo y ocupacional, de los solicitantes: Se trataba, en su mayoría, de trabajadoras del ámbito profesional correspondiente a esta cualificación y que contaban con un nivel formativo mínimo/en general de graduado escolar, además de cursos de actualización profesional.

Análisis de las expectativas formativas y ocupacionales de los solicitantes: Las expectativas fueron, sobre todo, ocupacionales, buscando el mantenimiento del puesto de trabajo; obteniendo para ello la acreditación de competencias para solicitar así el certificado de profesionalidad que exige la normativa anteriormente indicada para el año 2015.

Valoración global del proceso y resultados obtenidos: En general, se valoró positivamente el procedimiento, aunque se estableció que los resultados positivos habían dependido, sobre todo, de los años de experiencia de los candidatos, relacionada directamente con las actividades profesionales que se desarrollaban para cada una de las unidades de competencia.

\section{Opinión de candidatos ${ }^{19}$ :}

En relación a:

Motivación para participar en el PREAR: En su mayoría valoraban este proceso como interesante para obtener el certificado de profesionalidad que facilite la permanencia en su puesto de trabajo.

Vías de acceso a la convocatoria: La mayoría se informó de la convocatoria de este procedimiento en su propio centro de trabajo, a través de sus equipos directivos y compañeros de trabajo.

Valoración de la fase de asesoramiento: En general, se valoró como po-

19 Se entiende por candidatos a aquellos solicitantes que han sido seleccionados para su posterior participación en las diferentes fases del PREAR 
sitiva, ajustado a las necesidades de cada candidato, aunque a algunos candidatos (sobre todo de mayor edad) les costaba comprender la redacción de algunas preguntas y se hacía necesario adaptarlas a su nivel. También se daba el problema de que en muchas ocasiones no sabían contestar (oral o escrito) siguiendo los pasos o protocolo establecidos en el PREAR, porque estaban acostumbrados a practicar esas actividades diariamente en su puesto de trabajo con una terminología y protocolo propios de cada centro de trabajo.

Valoración de la fase de evaluación: La mayoría consideraba que tuvo algunas dificultades; como que las pruebas de evaluación no se ajustaban a situaciones reales de su profesión, tal y como la desarrollaban habitualmente en su centro de trabajo específico (con una metodología de trabajo que podía plantear diferencias en aspectos básicos).

Valoración de los resultados obtenidos: En general, se valoraron como positivos, aunque en una de las unidades de competencia consideran que han tenido mayores problemas y peores resultados, ya que incluye en sus contenidos aspectos más teóricos que el resto.

Expectativas laborales y formativas: La expectativa principal era la de obtener el certificado de profesionalidad para mantener su puesto de trabajo. Con este mismo fin muchos de los participantes se plantearon matricularse en el Ciclo Formativo de Grado Medio de Atención Sociosanitaria y cursar los módulos que les faltaban para obtener también el título y favorecer así la permanencia en el puesto de trabajo y la promoción laboral.

Valoración general del proceso y propuestas de mejora: Se valoró el proceso como bastante positivo y, en general, se consideró que les había ayudado a actualizar sus conocimientos y procedimientos, además de la utilidad para la acreditación de las competencias demostradas.

Respecto a la segunda cualificación: Atención sociosanitaria a personas en el domicilio ( $A S P D$ ), en la figura 2 se puede constatar un número de solicitantes alto (aunque un poco más reducido que la cualificación anterior). También se constata, respecto a la primera cualificación -ASPDI-, que un mayor número de candidatos acreditó todas las Unidades de Competencia. Del mismo modo, es destacable que la gran mayoría de candidatos fueron mujeres, aunque en esta cualificación es en la que se presentan, respecto a las demás el mayor número de hombres. 


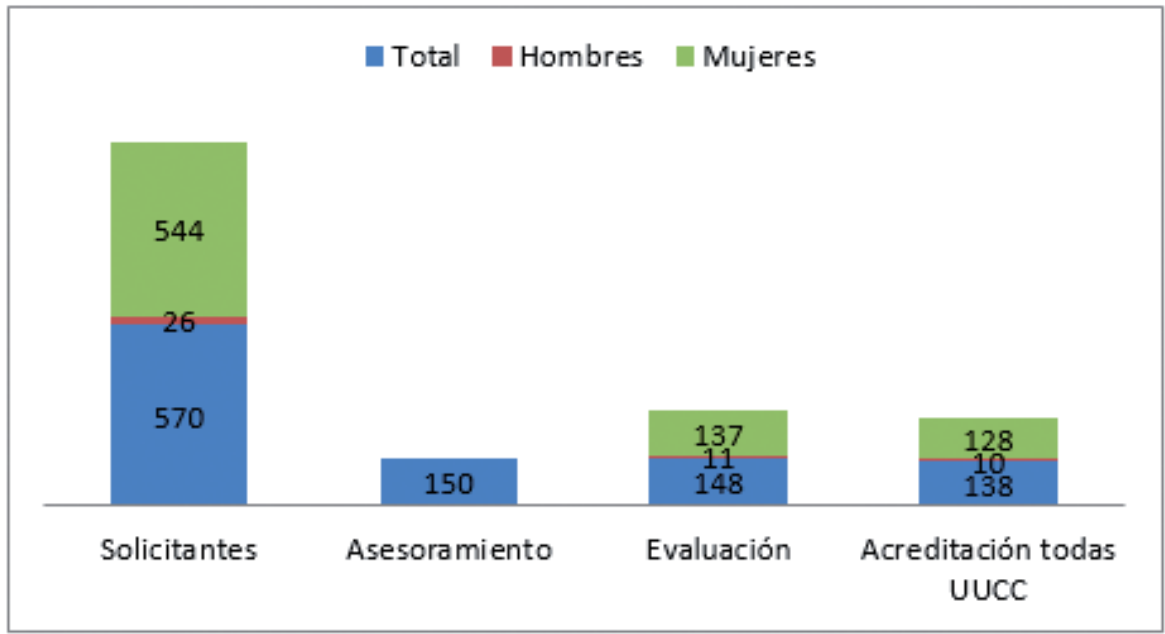

Figura 2. Participación en el PREAR para la cualificación de ASPD en la Región de Murcia. Convocatoria 2010.

Fuente: Elaboración propia a partir de la explotación de datos facilitada por el ICUAM.

\section{Resultados:}

Opinión de asesores/evaluadores:

En relación a:

Fase de habilitación como asesor/evaluador: En general se consideró que la formación ofrecida por la Consejería de Educación y Empleo debería haber sido más práctica; que dotara de instrumentos específicos de intervención para conocer mejor cómo desenvolverse como asesor/a o evaluador/a en el proceso. En el caso de las participantes que se han habilitado por el curso ofertado por los Ministerios de Trabajo y de Educación (fue realizado a través de la UNED) se consideró como un proceso formativo más completo y capacitador.

Desarrollo de los procesos de asesoramiento y de evaluación: En general, se valoró que el Procedimiento contó con un plazo demasiado limitado en principio, lo que había obligado a trabajar demasiadas horas por semana; pero que desde el propio ICUAM se fueron flexibilizando los plazos, facilitando así los procesos.

Se consideraba que había que trabajar con demasiada documentación administrativa, sobre todo en la fase de asesoramiento.

Los asesores/evaluadores participantes confirmaron que tuvieron una coordinación muy adecuada entre ellos mismos y así lograron realizar 
un mejor y más efectivo trabajo. Proponen que se establezca la figura de un coordinador dentro del grupo de asesores y también de éstos con evaluadores.

También consideraban que no se aprovechó totalmente en la fase de evaluación toda la información que se generó en el proceso de asesoramiento.

Se consideró importante que, al finalizar el proceso de asesoramiento, se orientara a los candidatos sobre cuáles son sus principales carencias en algunas de las competencias, para que buscaran la forma de compensarlas de cara a la evaluación. Con este fin los asesores ofrecieron a los candidatos información sobre recursos educativos y soportes didácticos para completar su formación en dichas competencias.

\section{Perfil de los solicitantes atendidos:}

El perfil dominante ha sido: mujer de 45 a 50 años, que lleva de 5 a 10 años trabajando como auxiliar de ayuda a domicilio para alguna empresa o similar. Con nivel formativo de graduado escolar y que vienen del ámbito de la empresa, mayoritariamente en activo.

Los asesores/evaluadores participantes también consideraban que la mayoría de candidatos daba mucha más importancia a la experiencia que a la formación de cara a la obtención de la acreditación de competencias.

Evaluación de los itinerarios, formativo y ocupacional, de los solicitantes: En general, se consideraba que los candidatos habían llegado a la ayuda a domicilio con una formación básica y que los ayuntamientos $u$ otras empresas asumían ese personal, incluso, más tarde, algunas de las empresas subcontrataban los servicios a los ayuntamientos. Respecto a la formación que habían recibido los candidatos, confirmaron que se trataba de formación continua que en muchos casos habían ofertado los propios ayuntamientos o las empresas, aunque en los últimos años dejaron de formarse considerando que no lo precisaban.

Evaluación de las expectativas formativas y ocupacionales de los solicitantes: Los asistentes consideraron que la motivación era mucho mayor en los candidatos que no se presentaban sólo para mantener su puesto de trabajo ya que en este último caso se consideraba más una imposición que una elección.

Valoración global del proceso y resultados obtenidos: Se valoró que, en general, el proceso fue adecuado aunque se tuvieron que prorrogar de- 
masiadas veces los plazos y eso hacía que los candidatos se sintieran desorientados. Respecto a resultados, consideraron positivo que la mayoría acreditaron todas las unidades de competencia, aunque tuvieron mayores dificultades en una de éstas cuyos contenidos eran de carácter más teórico. También consideraron que la experiencia era importante pero siempre que quedara vinculada a la puesta en práctica de las competencias reales de este perfil profesional.

Opinión de candidatos:

En relación a:

Motivación para participar en el PREAR: Principalmente los asistentes confirmaron que la motivación principal era la de mantener sus puestos de trabajo a partir del 2015.

Vías de acceso a la convocatoria: Les informaron las empresas y los propios ayuntamientos de los que dependen los servicios de ayuda a domicilio donde trabajaban.

Valoración de la fase de asesoramiento: En general se valoró como positiva, aunque algunos participantes consideraron que se debería haber orientado mejor a los candidatos dependiendo de las expectativas que mostraran (laborales o formativas).

Valoración de la fase de evaluación: Se confirmó una valoración positiva de los participantes respecto a esta fase; aunque coincidieron en que sería preferible que tanto en la entrevista inicial como en todas las pruebas de evaluación se hicieran demostraciones de forma procedimental y no, como a veces se les pidió, en que solamente explicaron cómo intervendrían ante una situación práctica.

Valoración de los resultados obtenidos: En general se consideraron adecuados los resultados que habían obtenido en el procedimiento.

Expectativas laborales y formativas: La mayoría confirmó que se había presentado al proceso para mantener su puesto de trabajo. Respecto a expectativas formativas, los participantes consideraron que para obtener el título de Grado Medio de Atención Sociosanitaria tras acreditar en el PREAR todas las unidades de competencia, tendrían que cursar demasiados módulos para completar dicha titulación.

Valoración general del proceso y propuestas de mejora: El proceso se valoró como excesivamente largo y se propuso que se simplificaran los trámites del mismo y se informara mejor sobre la convocatoria y calendarios de desarrollo. 
Respecto a la tercera cualificación: Educación Infantil, en la figura 3 se puede constatar que contó con el menor número de solicitantes de la convocatoria 2010 y que, a diferencia de las otras dos cualificaciones, no se ha vuelto a convocar en la Región de Murcia. También cabe mencionar que se trata de la cualificación en la que solamente un $16.7 \%$ de candidatos acreditó todas las Unidades de Competencia. En esta cualificación es en la que se presentan, respecto a las demás el menor número de hombres.

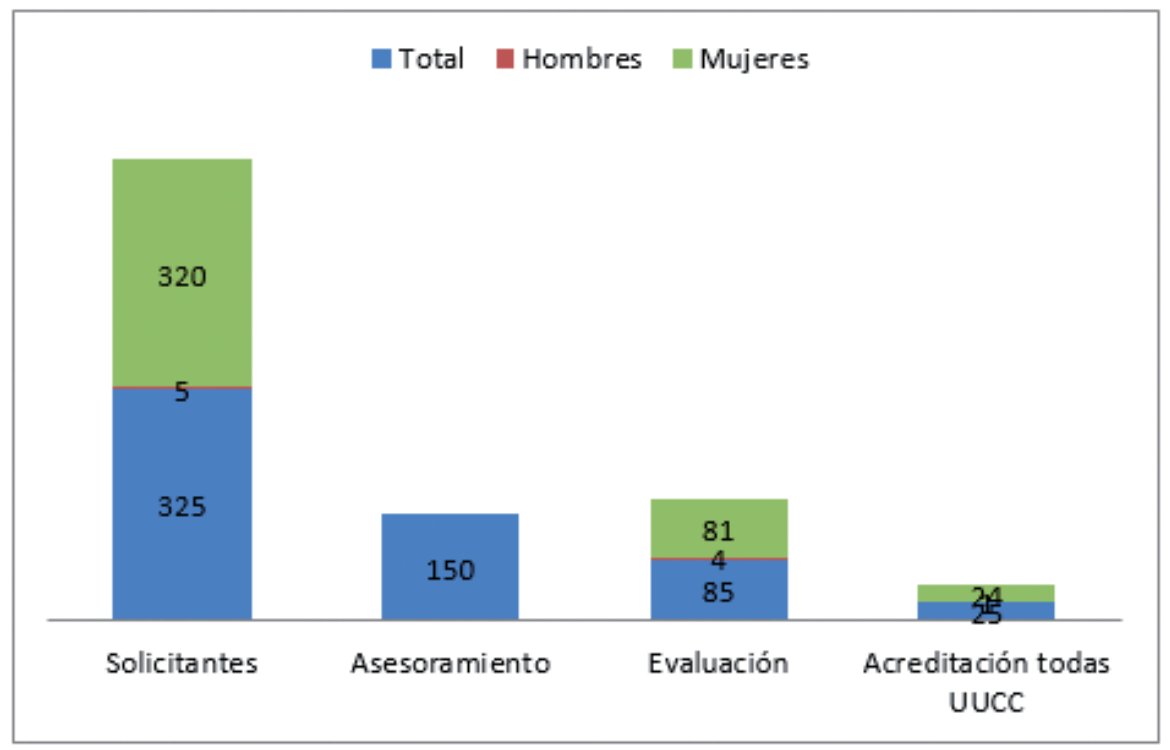

Figura 3. Participación en el PREAR para la cualificación de E.I. en la Región de Murcia. Convocatoria 2010

Fuente: Elaboración propia a partir de la explotación de datos facilitada por el ICUAM.

Opinión de asesores/evaluadores (datos basados en entrevistas personales). En relación a:

Fase de habilitación como asesor/evaluador: Se valoró como muy intenso y completo el proceso de habilitación seguido a través de la UNED por las entrevistadas (asesoras y evaluadoras).

Desarrollo de los procesos de asesoramiento y de evaluación: El número de candidatos asignado por habilitado se consideró excesivo en la fase de asesoramiento, aunque resultase más adecuado en la de evaluación ya que muchos candidatos no accedieron a esta segunda fase, pudiendo hacerlo, según la propia convocatoria del PREAR, sin necesidad previa de superar la fase de asesoramiento. 
Se estimó que el calendario se alargó demasiado, pero que fue necesario para un completo desarrollo del procedimiento.

Se consideró que los Ilamamientos para las citas en ambas fases, que finalmente fueron un máximo de cuatro (ya que la convocatoria establecía un mínimo de tres), habían contribuido a ralentizar el proceso y se debería reducir su número.

Cabe destacar que, según las entrevistadas, una minoría de los candidatos sólo poseía formación y ninguna experiencia. Estos candidatos fueron los que obtuvieron peores resultados en el procedimiento de asesoramiento.

Perfil de los solicitantes atendidos: El intervalo de edad de los candidatos fue muy amplio y la mayoría tenía formación de auxiliar de Jardín de Infancia.

Evaluación de los itinerarios formativo y ocupacional de los solicitantes: Se confirmó que, con mucha frecuencia, los candidatos que se presentaron habían trabajado durante los últimos años en Educación Infantil (sobre todo en escuelas infantiles).

Evaluación de las expectativas formativas y ocupacionales de los solicitantes: Se destacó que las expectativas de los candidatos se habían visto en parte frustradas ya que al final de este proceso no obtenían directamente el equivalente al título ni un certificado de profesionalidad, lo que motivó que muchos de los candidatos desistieran durante la fase de asesoramiento; incluso, algunos otros durante la fase de evaluación. Este desajuste se debió principalmente a la existencia de otras fuentes de información, diferentes a la oficial, a las que los candidatos asignaron también credibilidad en cuanto a las expectativas de certificación y titulación.

Valoración global del proceso y resultados obtenidos: En general se considera que el desarrollo de este proceso ha sido positivo; aunque como se alargó mucho más de lo previsto inicialmente, se planteó que se podría considerar la posibilidad de liberación de horario (en su trabajo habitual) de los asesores y evaluadores para acortar los plazos del procedimiento (tanto del asesoramiento como de la evaluación).

Se valoró que la mayor dificultad, al proceder los candidatos de un entorno profesional correspondiente a un nivel de cualificación 2 (jardín de infancia), fue que no demostraron nivel suficiente en las unidades de competencia más teóricas de esta cualificación que tiene un nivel 3, no acreditando las mismas muchos de los candidatos. 


\section{Opinión de candidatos:}

Motivación para participar en el PREAR: La mayoría de participantes coincidieron en que deseaban obtener una mayor especialización y reconocimiento de sus competencias para un mejor acceso al mercado de trabajo de esta cualificación de Educación Infantil.

Vías de acceso a la convocatoria: Las vías principales de información para acceder finalmente a esta convocatoria principalmente se facilitaron a los solicitantes a través de la propia Consejería de Educación a la que han acudido a informarse, o bien por medio de las agencias de desarrollo local y muy ocasionalmente fueron a través del SEF.

Valoración de la fase de asesoramiento: En general, se valoró como muy positivo el trabajo realizado por el personal asesor; sobre todo porque les facilitaba entender el contenido de las competencias que venían redactadas en lenguaje técnico.

También, había una percepción negativa de los candidatos en cuanto a que este proceso de asesoramiento se centró demasiado en los aspectos teóricos de las competencias más que los procedimientos que implican la práctica profesional.

Se subrayó por muchos candidatos que el asesoramiento se dilató demasiado en el tiempo y que se debería solamente haber asesorado sobre cómo prepararse de cara a la fase de evaluación; y sin embargo mantienen que también se evaluaron las competencias profesionales y de esta forma se solapaban funciones entre ambas fases.

Valoración de la fase de evaluación: En primer lugar, los participantes en el grupo de discusión coincidieron en que los malos resultados tras el informe de asesoramiento, con bastantes unidades de competencia que se veían obligados a prepararse de cara a la evaluación, motivaron un gran abandono del procedimiento ya que no encontraban fuentes de información adecuadas y muchos acudieron a Internet porque no tenían acceso a dichas fuentes.

La información recibida por los candidatos respecto al Plan Individual de Evaluación que se centraba en las Unidades de Competencia no demostradas hasta ese momento, se valoró por los participantes como mejorable ya que se envió por Internet y en varios casos no se accedió a la misma dentro de plazo para poder presentarse a las pruebas de evaluación de dichas competencias.

Respecto al informe de evaluación, se consideró que ha llegado demasiado tarde esa información ya que los que han querido preinscribirse 
en el Ciclo Formativo de Educación Infantil han tenido que hacerlo sin dicho informe, que ha llegado en la mayoría de casos más tarde.

También se valoró negativamente el hecho de no poder terminar en un mismo curso académico la formación de este Ciclo Formativo, ya que en muchos casos se han debido cursar módulos profesionales de primer y de segundo cursos.

Valoración de los resultados obtenidos: En general consideraron positivo el esfuerzo invertido de cara a los resultados obtenidos, aunque en muchos casos deseaban haber podido acreditar un número mayor de Unidades de Competencia.

También valoraron que tanto la experiencia como la formación son indispensables para un buen resultado en el procedimiento, pero destacaron que determinadas Unidades de Competencia (en las que peores resultados se ha tenido) precisaban de una mayor formación teórica.

Expectativas laborales y formativas: La mayoría destacó las dificultades para encontrar trabajo en este sector profesional y se decantaron por completar su formación accediendo al Ciclo Formativo de Grado Superior de Educación Infantil para obtener ese título de Técnico Superior. También valoraron negativamente que la única salida de este procedimiento para esta cualificación fuera la citada formación ya que no se expedía un certificado con efectos laborales al término del PREAR para la cualificación profesional de Educación Infantil.

Valoración general del proceso y propuestas de mejora: Se valoró en general como positivo aunque se contemplaron múltiples posibilidades de mejora como: una mejor coordinación entre el ICUAM y el SEF para dar información sobre la convocatoria del PREAR. También se consideraba necesario una mayor utilización de las sesiones grupales con los candidatos, sobre todo en la fase de asesoramiento, teniendo en cuenta también el factor de la proximidad para minimizar sus desplazamientos.

\section{Conclusiones}

Entendemos que los objetivos específicos de esta investigación se han cubierto en lo relativo a la forma en que se han valorado los procesos de asesoramiento y de evaluación seguidos con cada una de las tres cualificaciones convocadas. También se ha realizado una primera valoración general de resultados por cualificación, tanto desde una pers- 
pectiva cuantitativa (resultados en términos de frecuencia del número de candidatos por UUCC acreditadas dentro de cada cualificación) como cualitativa (opiniones de asesores/evaluadores y candidatos recogidas a través de los grupos de discusión y entrevistas).

Respecto a la eficacia del proceso cabe destacar que en las cualificaciones de nivel 2, casi la totalidad de candidatos han logrado acreditar todas las Unidades de Competencia (72\% en Atención sociosanitaria a personas dependientes en instituciones sociales y $92 \%$ en Atención sociosanitaria a personas en el domicilio); sin embargo en la cualificación Educación Infantil este porcentaje se reduce a un 16,7\% para la totalidad de UUCC acreditadas; siendo destacable que un 20,6\% ha logrado acreditar 5 UUCC, sobre un total de 7.

En cuanto a la distribución de candidatos por sexos, cabe destacar la presencia mayoritaria de candidatas en todo este procedimiento, superando el $90 \%$ de representatividad en las tres cualificaciones convocadas, ya que se trata de perfiles profesionales clásicamente muy feminizados en el mercado laboral español.

También se ha realizado una primera aproximación comparativa sobre la acreditación de competencias realizada por vías no formales de formación y experiencia laboral y cabe mencionar que en términos generales estos resultados, para las tres cualificaciones convocadas, se valoran como conseguidos a un mayor nivel por candidatos que cuentan en sus historiales laborales y formativos con mayor peso en experiencia laboral. Sin embargo, se ha constatado que en las unidades de competencia que cuentan con contenidos de carácter más teórico son en las que, a pesar de dicha experiencia, se ha contado con peores resultados, sobre todo en la cualificación de nivel 3.

En líneas generales, tanto el proceso de asesoramiento como el de evaluación, seguidos para cada una de las cualificaciones, se han valorado como ajustados a los estándares de eficiencia/eficacia predefinidos por la propia entidad convocante. Se han señalado diferentes propuestas de mejora en algunos de sus aspectos, sobre todo referentes a los calendarios de aplicación, que en muchas ocasiones, se han alargado, debido principalmente al elevado número de llamamientos mínimos a realizar para cada candidato; y también debido a la ratio de candidatos por asesor/a y evaluador/a que en ocasiones se ha considerado demasiado elevada.

La principal limitación del presente estudio es su alcance territorial que queda circunscrito a la Región de Murcia, por ello se estima conve- 
La evaluación de competencias profesionales adquiridas a través de la experiencia y vías no formales de formación Manuel Madrid García, Juan Carlos Solano lucas y Pedro Baños Páez

niente la realización de estudios comparativos con los procesos seguidos en otras Comunidades Autónomas, en cuanto al seguimiento y resultados de este Procedimiento para estas tres cualificaciones.

\section{Referencias bibliográficas}

Alaluf, M. y Stroobants, M. (1994). “¿Moviliza la competencia al obrero?». Revista Europea de Formación Profesional, 1, 46-55.

Alex, L. (1991). «Descripción y registro de las cualificaciones. El concepto de cualificación. Formación Profesional, 2, 23-27.

Baños Páez, P.; Madrid García, M.; Solano Lucas, J.C. y Benito Martínez, J. (2011). «La evaluación de aprendizajes y competencias adquiridas a través de la experiencia laboral y vías no formales de formación en la convocatoria 2010-2011 del PREAR para tres cualificaciones de la Familia Profesional de Servicios Socioculturales y a la Comunidad». En XII Simposio Internacional de Didáctica de las Ciencias Sociales. Murcia 12-15 Abril.

Boyatzis, R. E. (1982). The competent manager. Nueva York: John Wiley y Sons.

Becker, G. (1964). Human Capital: a theoretical and empirical analysis with special reference to education. Nueva York: Columbia UniversityPress.

Bunk, G.P. (1994). La transmisión de las competencias en la formación y el perfeccionamiento profesionales en la RFA. Revista Europea de Formación Profesional, 1, 8-14.

Collins, R. (1976). Conflic Sociology. Nueva York: AcademyPress.

Crompton, R. y Jones, G. (1984). White-collar proletariat. Londres: Macmillan.

Echeverría, B. (2002). Gestión de la competencia de acción profesional. Revista de Investigación Educativa, 20:1,7-42.

Gil Flores, J. (2007). La evaluación de competencias laborales. Educación XX1. 10, pp. 83-106

Harper, D. (1987). Working Knowledge: Skill and community in a small shop. Chicago: University of Chicago Press.

Homs, O. (2008). La formación profesional en España. Hacia la sociedad del conocimiento. Barcelona, Fundación La Caixa.

Jenson, J. (1989). "The talents of women, the skills of men: flexible specialization and women». En S. Wood (ed.). The transformation of work? Skill, flexibility and the labour process (pp. 247-265). Londres: Unwin Hyman.

Jones, B. y Wood, S. (1984). «Qualifications tacites, division du travail et nouvelles technologies». Sociologie du Travail, 26 (4): 407-420.

Köhler, H. D. y Martín Artiles, A. (2007): Manual de la Sociología del Trabajo y de las Relaciones Laborales. Madrid: Delta.

Le Boterf, G. (2001). Ingeniería de las competencias. Barcelona: Ediciones Gestión 2000. Madrid García, M. (2009). «La Reforma de la Formación Profesional en el Ciclo Formativo de Grado Superior de Educación Infantil en la Región de Murcia». En XIV Conferencia de Sociología de la Educación. Lleida 17-18 Septiembre. 
La evaluación de competencias profesionales adquiridas a través de la experiencia y vías no formales de formación

Manuel Madrid García, Juan Carlos Solano lucas y Pedro Baños Páez

Madrid García, M.; Solano Lucas, J.C. y Baños Páez, P. (2011). «El reconocimiento oficial de competencias profesionales adquiridas por experiencia y vías no formales de formación». En III Congreso anual de la Red Española de Política Social. Pamplona $24-26$ Noviembre.

Madrid García, M.; Solano Lucas, J.C. y Baños Páez, P. (2012). «La reforma del Ciclo Formativo de Grado Superior de Educación Infantil en la Región de Murcia». En I Congreso nacional de investigación e innovación en educación infantil y primaria. Murcia 22 -24 Marzo.

Olaz, A. (2011). «Una aproximación conceptual a la cualificación profesional desde una perspectiva competencial». En Papers, 96/2, 589-616.

Parkin, F. (1979). Marxism and class theory: a bourgeois critique. New York: Columbia University Press.

Planas, J.; J. F. Giret; G. Sala y J. Vincens (2000), "Skills market: dynamics and regulation", en P. Descy y M. Tessaring (eds.), Training in Europe. Second report on vocational training research in Europe, cedefop Reference series, Luxemburgo, Office for Official Publications of the European Communities, vol. II.

Planas, J. (2013), "El contrasentido de la enseñanza basada en competencias", en Revista Iberoamericana de Educación Superior (ries), México, unam-iisue/Universia, vol. iv, núm. 10, pp. 75-92, http://ries.universia.net/index.php/ries/article/view/289 [consulta: $18 / 10 / 2014]$.

Schultz, T.H. (1961). «Investment in human capital». American EconomicReview, 51, $1-17$.

Spencer, L. M. y Spencer. S. M.(1993). Competence at work. Models for superior performance. Nueva York: Wiley \& Sons.

Wood, S. (1981). The degradation of work: skill, de-skilling and the Braverman debate. Londres: Harper Collins.

Wood, S. (1987). «The Deskilling Debate. New Technology and Work Organization». Acta Sociológica, 30 (1): 3-24.

\section{Normativa:}

Ley Orgánica 5/2002 de las Cualificaciones y de la Formación Profesional. Publicada en: BOE $n^{\circ} .147$, de 20 de junio de 2002.

Real Decreto 496/2003, de 2 de mayo, por el que se establece el título de Técnico en Atención Sociosanitaria y las correspondientes enseñanzas comunes. Publicado en: BOE $n^{\circ} .124$, de 24 de mayo de 2003.

Real Decreto 1394/2007, de 29 de octubre, por el que se establece el título de Técnico Superior en Educación infantil y se fijan sus enseñanzas mínimas. Publicado en: BOE $\mathrm{n}^{\circ}$. 282, de 24 de noviembre de 2007.

Real Decreto 1224/2009, de 17 de julio, de reconocimiento de las competencias profesionales adquiridas por experiencia laboral. Publicado en: BOE n. 205, de 25 de agosto de 2009.

Orden 1/07/2010 de Convocatoria en la Región de Murcia del PREAR para las cualifica- 
La evaluación de competencias profesionales adquiridas a través de la experiencia y vías no formales de formación Manuel Madrid García, Juan Carlos Solano lucas y Pedro Baños Páez

ciones de: Atención sociosanitaria a personas dependientes en instituciones sociales, Atención sociosanitaria a personas dependientes en el domicilio y Educación Infantil. Publicada en: BORM nº 167 Jueves, 22 de julio de 2010.

\section{Webgrafía}

Instituto Nacional de las Cualificaciones. Datos recogidos sobre el Procedimiento de Reconocimiento de Competencias adquiridas por experiencia laboral. Agosto 2013. http://www.educacion.gob.es/educa/incual/pdf/Acreditacion/Inf Datos Proc Acred 2013 agosto.pdf. [consulta: 18/10/2014].

Instituto de las Cualificaciones de la Región de Murcia. Resultados y estadísticas de las convocatorias PREAR 2010, 2012, 2013. http://www.icuam.es/web/guest/91. [consulta: 18/10/2014]. 
